\title{
ELECTROPHORESIS
}

\section{Validation of CE modelling with a contactless conductivity array detector}

\begin{tabular}{|r|l|}
\hline Journal: & ELECTROPHORESIS \\
\hline Manuscript ID & elps.201500424.R1 \\
\hline Wiley - Manuscript type: & Research Paper \\
\hline Date Submitted by the Author: & 13-Jan-2016 \\
\hline Complete List of Authors: & $\begin{array}{l}\text { Thormann, Wolfgang; University of Bern, Clinical Pharmacology Laboratory } \\
\text { Hauser, Peter; The University of Basel, Department of Chemistry }\end{array}$ \\
\hline Keywords: & $\begin{array}{l}\text { capillary electrophoresis, computer simulation, array detector, } \\
\text { electroosmosis, LPA coated capillary }\end{array}$ \\
\hline
\end{tabular}

SCHOLARONE ${ }^{\text {"m }}$

Manuscripts 


\title{
Validation of CE modelling with a contactless conductivity array detector
}

\author{
Jitka Caslavska $^{1}$, Israel Joel Koenka ${ }^{2}$, Peter C. Hauser ${ }^{2}$, Wolfgang Thormann ${ }^{1, *}$ \\ ${ }^{1}$ Clinical Pharmacology Laboratory, Institute for Infectious Diseases, University of Bern, \\ Murtenstrasse 35, 3008 Bern, Switzerland. \\ ${ }^{2}$ Department of Chemistry, University of Basel, Spitalstrasse 51, 4056 Basel, Switzerland.
}

Short title: CE in LPA coated capillaries

Keywords: array detector, capillary electrophoresis, computer simulation, electroosmosis, LPA coated capillary, isotachophoresis

\author{
Abbreviations: $\mathrm{C}^{4} \mathrm{D}$, contactless conductivity detector; HV, high voltage; LE, leading electrolyte; \\ LPA, linear polyacrylamide; NPE, norpseudoephedrine; PFA, perfluoroalkoxy alkane; SIA, \\ sequential injection analysis, TE, terminating electrolyte; \\ *Corresponding author: \\ Prof. Dr. W. Thormann \\ Clinical Pharmacology Laboratory, Institute for Infectious Diseases \\ University of Bern \\ Murtenstrasse 35 \\ phone: ...41316323288 \\ fax: ..4131632 4997 \\ CH - 3008 Bern, Switzerland \\ email: wolfgang.thormann@ifik.unibe.ch
}




\begin{abstract}
Dynamic computer simulation data are compared for the first time with CE data obtained with a laboratory made system comprising an array of 8 contactless conductivity detectors $\left(\mathrm{C}^{4} \mathrm{Ds}\right)$. The experimental setup featured a $50 \mu \mathrm{m}$ I.D. linear polyacrylamide (LPA) coated fused-silica capillary of $70 \mathrm{~cm}$ length and a purpose built sequential injection analysis manifold for fluid handling of continuous or discontinuous buffer configurations and sample injection. The LPA coated capillary exhibits a low EOF and the manifold allows the placement of the first detector at about $2.7 \mathrm{~cm}$ from the sample inlet. Agreement of simulated electropherograms with experimental data was obtained for the migration and separation of cationic and anionic analyte and system zones in CZE configurations in which EOF and other column properties are constant. For configurations with discontinuous buffer systems, including ITP, experimental data obtained with the array detector revealed that the EOF is not constant. Comparison of simulation and experimental data of ITP systems provided the insight that the EOF can be estimated with an ionic strength dependent model similar to that previously used to describe EOF in fused-silica capillaries dynamically double coated with Polybrene and poly(vinylsulfonate). For the LPA coated capillaries, the electroosmotic mobility was determined to be 17 -fold smaller compared to the case with the charged double coating. Simulation and array detection provide means for quickly investigating electrophoretic transport and separation properties. Without realistic input parameters, modelling alone is not providing data that match CE results.
\end{abstract}




\section{Introduction}

In a recent publication, the construction of a CE system featuring an array of 16 contactless conductivity detectors $\left(\mathrm{C}^{4} \mathrm{Ds}\right)$ was reported [1]. In that instrument, the detectors were arranged along $70 \mathrm{~cm}$ length of a capillary with $100 \mathrm{~cm}$ total length and the capillary was interfaced to a setup with a sequential injection analysis (SIA) manifold for automation of fluid handling. The new system allows the simultaneous monitoring of electrophoretic processes throughout a large part of the separation channel and its use was demonstrated via following of the progress of the separation of simple ions by zone electrophoresis [1]. Monitoring of the conductivity or electric field strength at multiple locations along the capillary can be considered universal for electrophoresis as it visualizes changes in ionic composition and thereby provides a tool to study the dynamics of boundaries, peaks and zones in all electrophoretic modes, including moving boundary electrophoresis, isotachophoresis, zone electrophoresis and isoelectric focusing [2-6]. Different types of conductivity detectors for capillary electrophoresis were developed over the years, the most appealing being those based on capacitively coupled contactless sensing, an approach which is not subject to corrosion and other deleterious surface phenomena which can occur at electrodes in contact with a liquid medium [7-12]. The first contactless conductivity detector was built for zone detection in capillary isotachophoresis [13].

The elucidation of the dynamics of electrophoretic separations and processes is of high interest to explore and understand the basic phenomena occurring under the influence of the electric field applied to a liquid medium and to optimize configurations for electrophoretic analyses. Dynamic changes along the electrophoretic column can be investigated experimentally using conductivity [1], electric field strength [2-6] or optical [14-18] detection principles, or by computer simulation [1928]. Dynamic simulation provides plentiful data for any given electrophoretic system, namely the separation dynamics of sample components together with the ongoing changes of buffer components, $\mathrm{pH}$, ionic strength, conductivity and electric field strength along the separation column, and effects of fluid flow, including electroosmosis. Comprehensive simulators, including GENTRANS [19-22], SIMUL5 [23,24] and SPRESSO [25], are available for that task. They are one-dimensional transient electrophoresis models which allow high-resolution, real-power simulations for any electrolyte arrangements, including those associated with zone electrophoresis, isotachophoresis and isoelectric focusing [26-28].

$\mathrm{CE}$ is typically associated with fluid flow, mostly electroosmosis. Imposed hydrodynamic flow is used in special applications [29]. The EOF is an electrokinetic phenomenon associated with the surface charge at the inner capillary wall and the composition of the solution, including $\mathrm{pH}$ and 
ionic strength. GENTRANS permits the combined simulation of the temporal behavior of electrophoresis and electroosmosis under constant voltage or current conditions and was previously used to estimate the $\mathrm{pH}$ and ionic strength dependent EOF in uncoated fused-silica $[21,30]$ and polymethylmethacrylate (PMMA) capillaries [30]. In another effort, the $\mathrm{pH}$ independent EOF in dynamically double coated fused-silica capillaries featuring a charged surface was studied in the same way [31]. Furthermore, this dynamic capillary electrophoresis model which uses material or surface property specific input data for estimation of electroosmosis was applied to investigate fundamental aspects of isoelectric focusing in capillaries or microchannels made from bare fusedsilica, fused-silica coated with a sulfonated polymer, PMMA and poly(dimethylsiloxane) [32]. In GENTRANS, EOF is treated as plug flow with no contribution to dispersion, whereas the impact on zone and boundary dispersion caused by hydrodynamic flow is calculated with the Taylor-Aris equation [29].

The main focus of this paper is the validation of computer simulation data generated by GENTRANS with a CE setup featuring $8 C^{4}$ Ds along a $50 \mu \mathrm{m}$ I.D. fused-silica capillary coated with linear polyacrylamide (LPA), an approach that is reported here for the first time. The previously described laboratory made $\mathrm{CE}$ system with $\mathrm{C}^{4} \mathrm{D}$ array detection [1] was modified to allow (i) application of hydrodynamic flow during an electrophoretic run and (ii) handling of configurations with continuous and discontinuous buffer systems. The changes in the experimental setup are described and computer predicted electropherograms using the GENTRANS software are compared to those obtained experimentally. Examples studied include i) the migration and separation dynamics of cationic and anionic analyte and system zones in CZE with imposed hydrodynamic buffer flow, and ii) the following of electrophoretic boundaries formed in a discontinuous buffer system and of an ITP sample zone in configurations without pressure driven hydrodynamic flow. The setup used allows the placement of $\mathrm{C}^{4} \mathrm{Ds}$ detectors close to the sample inlet such that comparison of experimental and simulation data can be employed to characterize transient zone patterns formed around the sampling compartment of the capillary and the temporal behavior of EOF in an LPA coated capillary under ITP conditions.

\section{Materials and methods}

\subsection{Reagents}

If not stated otherwise, all chemicals used were of analytical or research grade. Sodium acetate anhydrous, DL-mandelic acid, nicotinic acid, glycolic acid and L-arginine were purchased from Fluka (Buchs, Switzerland). Acetic acid, formic acid, sodium hydroxide solution Titripur $0.1 \mathrm{~mol} / \mathrm{L}$ and benzoic acid were from Merck (Darmstadt, Germany). (1S,2S)-(+)-norpseudoephedrine 
(+NPE), (1R,2R)-(-)-norpseudoephedrine (-NPE) and tryptamine were from Sigma-Aldrich (St. Louis, MO, USA).

\subsection{Instrumental setup}

An overall schematic representation of the CE instrument is depicted in Fig. 1. The setting comprises a pressure assisted SIA system [1,33-37] designed to deliver solutions to the capillary inlet and into it using hydrodynamic pressure. The heart of the system is a syringe pump with a 3way valve (Cavro XLP 6000, Tecan, Crailsheim, Germany) which is connected to a 6-port channel selection valve (Cavro Smart Valve, Tecan) via a $400 \mu \mathrm{L}$ holding coil. The holding coil's volume was set to be much larger than the volume between the selection valve and the capillary entrance $(<10 \mu \mathrm{L})$. All tubes of the system are made of perfluoroalkoxy alkane (PFA) and have a 1/16" O.D. (Idex, Wertheim-Mondfeld, Germany). One of the 3-way valve ports is connected to solution $\mathrm{S}_{0}$, which is the only solution allowed in the syringe pump. Solutions $S_{1}$ to $S_{5}$ are connected to ports 1 to 5 of the selection valve and are transferred to the capillary entrance via use of the holding coil. For CZE, $\mathrm{S}_{0}$ was the BGE and $\mathrm{S}_{5}$ was the sample solution. For ITP, $\mathrm{S}_{0}$ was the terminating electrolyte (TE), $\mathrm{S}_{5}$ was the leading electrolyte (LE) and $\mathrm{S}_{4}$ was the sample. Port 6 of the selection valve is connected to an injection interface to which the CE capillary and the ground electrode are connected. The injection interface was built by connecting two $1 / 16$ " T-interconnects made from solvent resistant ULTEM resin (T116-203, LabSmith, Livermore CA, USA) with a short 0.04" I.D. PFA tubing in between. One T-interconnect was used as is (inner channels of 0.02 ") and one was machined to have wider channels (0.06") such that the fluid volume around the electrode is large enough to avoid the interference of electrolysis products with the CE separation.

All tubes upstream to the capillary are 0.01 " I.D., while all tubes downstream and the second T to which the ground electrode is connected are 0.04" I.D. (thin and thick lines in Fig. 1, respectively). This reduces post-capillary flow resistance in order to minimize undesired diversion of solutions into a capillary when passing by its inlet. Furthermore, the injection interface is connected to a 3way valve (AV201-T116, LabSmith) which permits selection of the waste container (W) or one of two pressure regulators ( $\mathrm{P}_{\text {high }}$ or $\mathrm{P}_{\text {low }}$, controlled by another 3-way valve (AV201-T116, LabSmith)). The high pressure regulator can provide pressures between 10-100 psi (VSO-BT, Parker Hannifin, Etoy, Switzerland) and is used to flush the capillary between separations, while the low pressure regulator can provide pressures between 0.2-2 psi (OEM-EP, Parker Hannifin), which is used for sample plug injection and for applying hydrodynamic co-flow during a separation. Both regulators are fed from a pressurized 4.5 Nitrogen cylinder (PanGas, Dagmersellen, Switzerland). The separation capillary used was a $70 \mathrm{~cm}$ long, $50 \mu \mathrm{m}$ I.D. fused-silica capillary coated with LPA 
(Polymicro Technologies, Phoenix, AZ, USA), and was passed through 8 purpose-made $\mathrm{C}^{4} \mathrm{Ds}$ [1]. The centers of the 8 detectors were positioned 2.7, 8.2, 13.2, 18.4, 23.5, 28.5, 33.5 and $38.5 \mathrm{~cm}$ away from the capillary's injection end (distance between detectors was about $5 \mathrm{~cm}$ ). The capillary end was dipped into a $50 \mathrm{~mL}$ plastic vial (Falcon tube, Corning, Amsterdam, The Netherlands) containing the outlet solution $\mathrm{S}_{\text {out }}$ (BGE for CZE separations and LE for ITP) and a stainless steel electrode which was connected to a dual polarity $\pm 30 \mathrm{kV}$ supply (CZE2000, Spellman, Pulborough, UK). As a precaution, the outlet container was placed in a Perspex safety box equipped with a micro switch to disrupt high voltage (HV) on opening. All system parts (syringe pump, valves, pressure regulators and HV supply) were connected to a purpose made control box and were operated from a PC. The control box included an Arduino Nano 3.0 microcontroller (Gravitech, Minden, NV, USA) which was tethered to a purpose made GUI software on the PC based on the open-source framework Instrumentino [38,39]. This enabled to control each experiment and easily execute long run sequences for optimizing experimental parameters such as sample plug injection conditions. Detector data were recorded with a 16-channel e-corder (eDAQ, Denistone East, NSW, Australia) and processed with the Chart software package (eDAQ). Applied voltage and current as function of time were monitored by Instrumentino. The current reading provided a noisy signal which was smoothed by regression analysis.

\subsection{Experimental procedures}

New capillaries were rinsed with water, $0.1 \mathrm{M} \mathrm{NaOH}$ and BGE or LE (for a few minutes each). Between runs capillaries were rinsed with BGE for CZE and with LE in the case of ITP. Overnight or when not in use, capillaries were stored filled with 70\% isopropanol. Each experiment was executed by a sequence of system-defined actions comprising i) initialization, ii) interface flushing, iii) capillary flushing, iv) sample injection and v) application of voltage with or without co-flow. For CZE separations, the interface was flushed with $400 \mu \mathrm{L}$ of BGE, followed by a capillary flush for 3 min using 20 psi. The sample was injected at 1.16 psi for $12 \mathrm{~s}$. The interface was flushed again with $400 \mu \mathrm{L}$ of BGE followed by application of a constant $20 \mathrm{kV}$ using a co-flow produced by application of pressure between 1 and 2 psi. For ITP, the interface was flushed with $400 \mu \mathrm{L}$ of LE. This flush was performed 3 times, with short capillary flushes (10 s) in between, to ensure that any TE left from the previous run was completely removed. The cleaning progress was verified by checking the conductivity level which had to reach the expected level for pure LE. After this initial washing procedure, the capillary was flushed with LE for 3 min using 20 psi. Sample (or LE in case of blank runs) was introduced by application of 1.6 psi for $13 \mathrm{~s}$. The interface was flushed with 600 
$\mu \mathrm{L}$ of TE followed by application of constant voltage of $20 \mathrm{kV}$ or constant current of about $3 \mu \mathrm{A}$. No imposed co-flow was applied.

For measuring the low EOF and the imposed flow generated via application of pressure during electrophoresis in an LPA coated capillary, a setup with a $C^{4} D$ as described elsewhere [29,31] was employed. Briefly, it comprised a PrinCE sampler (model 560, Prince Technologies, Emmen, The Netherlands), a $50 \mu \mathrm{m}$ I.D. capillary of $90 \mathrm{~cm}$ total length and the detector at $47 \%$ of capillary length. BGEs of $10 \mathrm{mM}$ ionic strength, a sample composed of arginine and benzoic acid (1 $\mathrm{mM}$ each in $50 \%$ BGE, hydrodynamically injected at anodic capillary end) and a voltage of $20 \mathrm{kV}$ were applied. The temperature was ambient (around $24^{\circ} \mathrm{C}$ ). Flow properties were assessed by variation of applied pressure between 0 and $100 \mathrm{mbar}(0-1.45 \mathrm{psi})$ at a 20 mbar interval and the detected nonmigrating system peak (referred to as EOF or flow peak in case of absence and presence of imposed flow, respectively) was used for data evaluation. Detector data were collected at a sampling rate of $100 \mathrm{~Hz}$ and stored using an e-corder 401 (eDAQ, Denistone East, NSW, Australia) as described elsewhere [29].

\subsection{Computer simulations with GENTRANS and data presentation}

GENTRANS was executed on Windows 7 based PCs featuring i5 processors running at $2.4 \mathrm{GHz}$. Configurations under normal polarity with or without an imposed buffer flow towards the cathode were investigated. Electroosmosis was calculated as function of ionic strength according to the model described for fused-silica capillaries that were dynamically double coated with Polybrene and poly(vinylsulfonate) [31] and having an electroosmotic mobility determined for an LPA coated capillary. The component's input data for simulation are summarized in Table 1. If not stated otherwise, the impact of ionic strength on electrophoretic mobilities was not considered. For CZE simulations with imposed flow, the program featuring Taylor-Aris dispersion was used [29]. A 35 cm capillary divided into 35000 segments of equal length $(\Delta \mathrm{x}=10.0 \mu \mathrm{m})$ and a constant voltage of $5000 \mathrm{~V}(80 \mathrm{~V} / \mathrm{cm})$ were used. For constructing detector plots, simulation data storage occurred at 2 Hz. Simulations for $12.5 \mathrm{~min}$ of electrophoresis could thereby be executed in about $4 \mathrm{~h}$. Samples were applied at the anodic capillary end as a plug with a length of $1.0 \%$ of column length (initially placed between 1.0 and $2.0 \%$ of column length) and a boundary width of $0.001 \%$. The composition of the BGE and sample is given in the description of each example. For ITP simulations, a $17.5 \mathrm{~cm}$ separation space divided into 35000 segments of equal length $(\Delta \mathrm{x}=5.0 \mu \mathrm{m})$ and a constant current density of $370 \mathrm{~A} / \mathrm{m}^{2}$ or a constant voltage of $1500 \mathrm{~V}$ were employed. Sample was applied at the anodic capillary end as a plug with a length of $2.5 \%$ of column length (initially placed between 5.0 and $7.5 \%$ of column length). For $10 \mathrm{~min}$ of electrophoresis, this required 
simulation times between 4 and $9 \mathrm{~h}$. The composition of LE and TE are given in the description of each example. All simulations were executed with data smoothing as described elsewhere [28].

\subsection{Additional simulation tools and data presentation}

CZE simulation data produced with GENTRANS were compared to those predicted using PeakMaster 5.2 software [40] which can be downloaded for free from http://www.natur.cuni.cz/Gas. Sample plug lengths and the velocity of the imposed applied flow were estimated with the CE Expert Lite software (Beckman Coulter, Fullerton, CA, USA). For smoothing of experimental current data and making plots of experimental and simulation electropherograms, data were imported into SigmaPlot 12.5 (Systat Software, San Jose, CA, USA).

\section{Results and discussion}

\subsection{Electroosmosis and applied hydrodynamic flow in an LPA coated capillary}

In this paper, simulation data are compared to those obtained experimentally in LPA coated fusedsilica capillaries. For CZE, hydrodynamic flow was applied in order to be able to detect cationic and anionic zones in one run. For the simulation of electrophoretic processes in capillaries, it is necessary that EOF and applied hydrodynamic flow are known. Thus, using a conventional CE setup with conductivity detection [29], EOF and hydrodynamic flow as function of applied pressure in an LPA coated $50 \mu \mathrm{m}$ I.D. capillary were determined first. BGEs with ionic strength of 10.02 $\mathrm{mM}$ comprising $12 \mathrm{mM}$ HIBA and $10 \mathrm{mM} \mathrm{NaOH}$ (measured $\mathrm{pH} 4.66$ ) or $20 \mathrm{mM}$ nicotinic acid and $10 \mathrm{mM} \mathrm{NaOH}$ (measured pH 4.76) were employed. For the two buffers, evaluation of the velocity of the non-migrating system peak as function of applied pressure revealed linear relationships $\left(\mathrm{R}^{2}\right.$ of 0.9999 and 0.9996 , respectively) with the y-intercept reflecting EOF $(53.7$ and $52.2 \mu \mathrm{m} / \mathrm{s}$, respectively). The electroosmotic mobilities could thereby be calculated as $2.42 \times 10^{-9}$ and $2.35 \mathrm{x}$ $10^{-9} \mathrm{~m}^{2} / \mathrm{Vs}$, respectively, values that are considerably smaller than those observed in uncoated capillaries at that $\mathrm{pH}$ (about $25 \times 10^{-9} \mathrm{~m}^{2} / \mathrm{Vs}$ ) and capillaries dynamically double coated with Polybrene and poly(vinylsulfonate) (about $41 \times 10^{-9} \mathrm{~m}^{2} / \mathrm{Vs}$ ) [31]. For LPA coated capillaries and buffer $\mathrm{pH}<8$, the EOF is assumed to be essentially independent of $\mathrm{pH}$ [41]. Thus, EOF as function of ionic strength was estimated according to the model described for fused-silica capillaries dynamically double coated with Polybrene and poly(vinylsulfonate) via use of an electroosmotic mobility of $2.4 \times 10^{-9} \mathrm{~m}^{2} / \mathrm{Vs}$ instead of $41.1 \times 10^{-9} \mathrm{~m}^{2} / \mathrm{Vs}$. The slopes of the linear relationships revealed the imposed flow velocity per unit pressure (9.83 and $9.89 \mu \mathrm{m} / \mathrm{mbar}$, respectively). For a pressure of $80 \mathrm{mbar}(1.16 \mathrm{psi}$ ), the flow was about $788 \mu \mathrm{m} / \mathrm{s}$, a value that compares well to 780 $\mu \mathrm{m} / \mathrm{s}$ estimated with the CE Expert Lite calculator for a temperature of $25^{\circ} \mathrm{C}$. The CE Expert Lite 
calculator is based on the Poiseuille equation which describes how fluid under pressure is flowing through a cylindrical vessel. Experiments with array detection were made with a capillary of $70 \mathrm{~cm}$ total length. Corresponding flow values calculated for 25 and $30^{\circ} \mathrm{C}$ are 1000 and $1120 \mu \mathrm{m} / \mathrm{s}$, respectively. Thus, as the intracapillary temperature was assumed to be slightly above room temperature, the imposed pressure driven flow in our experiments at $1.16 \mathrm{psi}$ ( 80 mbar) was taken as $1050 \mu \mathrm{m} / \mathrm{s}$ and EOF was estimated by subtraction of $1050 \mu \mathrm{m} / \mathrm{s}$ from the total flow value which was determined from the flow peak in the experimental data.

\subsection{Capillary zone electrophoresis with array detection}

CZE in an LPA coated capillary was studied in order to validate the electrokinetic simulation model for configurations with constant column properties, including the small EOF encountered in this type of capillary. The example studied comprised a two-component BGE composed of $20 \mathrm{mM}$ formic acid and $10 \mathrm{mM} \mathrm{NaOH}$ together with four sample components (two cationic, two anionic) in a $70 \mathrm{~cm}$ long, $50 \mu \mathrm{m}$ I.D. fused-silica capillary whose inside wall was coated with LPA. Ionic strength, conductivity and $\mathrm{pH}$ of the BGE were calculated to be $10.17 \mathrm{mM}, 0.112 \mathrm{~S} / \mathrm{m}$ and 3.77, respectively. The sample was applied at the anodic capillary end and was transported along the capillary by the concomitant actions of electromigration, electroosmosis and hydrodynamic flow. According to the prediction of the PeakMaster software, this configuration produces two system peaks. The first one is migrating towards the cathode (calculated mobility: $12.00 \times 10^{-9} \mathrm{~m}^{2} / \mathrm{Vs}$ without correction of ionic strength, $13.53 \times 10^{-9} \mathrm{~m}^{2} / \mathrm{Vs}$ with correction of ionic strength), the second does not migrate and is thus also referred to as the EOF peak (or flow peak in case of a configuration with hydrodynamic flow). Both system peaks are characterized by a conductivity change and are thus recognizable with a conductivity detector. The sample was composed of benzoic acid, tryptamine, glycolic acid and L-arginine (1 mM each in BGE).

Simulation data generated with GENTRANS and application of Taylor-Aris diffusivity to account for dispersion caused by the imposed flow are depicted in Fig. 2A. As was discussed in a previous paper [29], electropherograms can be properly predicted by dynamic simulation using half of the column length together with half of imposed flow and current density (quarter of applied voltage). The time scales of predicted and experimental electropherograms are thereby identical but simulation time is much shorter compared to simulations with the full capillary length. Thus, simulations were performed with a $35 \mathrm{~cm}$ capillary (35000 segments, $\Delta \mathrm{x}=10.0 \mu \mathrm{m})$ at a constant $5000 \mathrm{~V}$, an imposed hydrodynamic flow of $525 \mu \mathrm{m} / \mathrm{s}$ towards the cathode and a sample occupying 1 $\%$ of capillary length at the anodic capillary end (placed between 1 and $2 \%$ of the capillary length with boundary widths of 0.001 ). Initial current density and EOF were calculated to be $1597.19 \mathrm{~A} / \mathrm{m}^{2}$ 
and $44.12 \mu \mathrm{m} / \mathrm{s}$, respectively. The execution time was $4.17 \mathrm{~h}$. The simulated electropherograms of all 8 detectors obtained with a sampling frequency of $2 \mathrm{~Hz}$ are presented in Fig. 2A. The selected positions for detectors 1 to 8 were $4.86,12.71,19.86,27.29,34.57,41.71,48.86$ and $56.00 \%$ of simulation column length, respectively, and correspond to those used in the experimental setup with the adjustment for the $1 \%$ initial shift of the sample position used in the simulation. The predicted detector data reveal that all four sample components provide zones which have a lower conductivity compared to the conductivity of the BGE. The opposite is true for the two system peaks.

Furthermore, the two anionic sample components (glycolic acid and benzoic acid) and the two system peaks are quickly separated (see signal of second detector). On the other hand, the two cationic sample components separate slowly and are not completely separated when they are transported across the last detector (top graph in Fig. 2A). In CZE configurations analyte and system peaks are the only discontinuities present. Thus, transport properties through the capillary should essentially be constant. The dynamic simulation approach of GENTRANS revealed this behavior. The computer predicted current density at $12 \mathrm{~min}$ is $1594.57 \mathrm{~A} / \mathrm{m}^{2}$ which is almost identical to that at the beginning of the run. The net buffer flow (sum of imposed flow and EOF) remains constant which indicates that the predicted EOF is constant. Both flows correspond to those monitored experimentally in the $70 \mathrm{~cm}$ capillary (see below).

For experimental validation, the sample was injected for $12 \mathrm{~s}$ at $1.16 \mathrm{psi}$ (80 mbar) thereby occupying a capillary segment of about $1 \%$ of capillary length (calculated with CE Expert Lite software). After sample application, the interface was flushed with $400 \mu \mathrm{L}$ of BGE followed by application of $20 \mathrm{kV}$ and a co-flow produced by a pressure of $1.16 \mathrm{psi}$ which generated a constant imposed flow towards the cathode $(1050 \mu \mathrm{m} / \mathrm{s}$, see Section 3.1). Conductivity electropherograms obtained with the 8 detectors are presented in Fig. 2B and compare well with those predicted by simulation shown in Fig. 2A. Electropherograms of the $8 \mathrm{C}^{4} \mathrm{Ds}$ were not normalized to the response of a selected detector. It is apparent that simulation is correctly predicting analyte and system peaks. Evaluation of the detector position for detectors 2 to 8 as function of the detection time of the flow peak (peak S2 in Fig. 2A) revealed a perfect linear relationship $\left(\mathrm{r}^{2}=1.000\right)$ with a slope representing the total flow $(1139 \mu \mathrm{m} / \mathrm{s})$ and an intercept $(2.56 \mathrm{~mm})$ indicating the imprecision of detector mounting in relation to the beginning of the capillary and placement of the initial sample plug. These data indicate that the EOF was $89.0 \mu \mathrm{m} / \mathrm{s}$ which corresponds to an electroosmotic mobility of $3.12 \times 10^{-9} \mathrm{~m}^{2} / \mathrm{Vs}$, a value that is comparable to that obtained during flow calibration experiments reported in Section 3.1. Measured voltage was constant throughout the experiment and the current was $6.7 \mu \mathrm{A}$ which compares well to that predicted for a $50 \mu \mathrm{m}$ ID capillary of $70 \mathrm{~cm}$ length at $20 \mathrm{kV}(6.27 \mu \mathrm{A})$. The applied power level was low $(0.193 \mathrm{~W} / \mathrm{m})$. 
The characteristics of system peaks are known to be dependent on buffer concentration and composition [42]. Thus, in order to visualize this aspect, the same sample was also analyzed in the BGE with half the concentration of the two buffer components $(10 \mathrm{mM}$ formic acid and $5 \mathrm{mM}$ $\mathrm{NaOH}$ ) and otherwise identical conditions. Ionic strength, conductivity and $\mathrm{pH}$ of the BGE were calculated to be $5.17 \mathrm{mM}, 0.0590 \mathrm{~S} / \mathrm{m}$ and 3.78, respectively. According to the prediction of the PeakMaster software, this configuration produces two system peaks. The first one is migrating towards the cathode (calculated mobility: $21.56 \times 10^{-9} \mathrm{~m}^{2} / \mathrm{Vs}$ without correction of ionic strength, $23.27 \times 10^{-9} \mathrm{~m}^{2} / \mathrm{Vs}$ with correction of ionic strength), the second does not migrate and is thus also referred to as the flow peak. Dilution of the BGE compared to that used for Fig. 2 has an impact on the migration velocity of system peak S1. Its mobility becomes higher and the system peak is predicted to interfere with the two cationic sample ions. This is nicely seen in the GENTRANS simulation data presented in Fig. 3A. Tryptamine and arginine do not separate properly and system peak $\mathrm{S} 1$ is broader compared to the data of Fig. 2A. The same behavior could be verified experimentally (Fig. 3B). Separation of the two anionic sample components (benzoic acid and glycolic acid) are unaffected by the buffer change. Both simulation and experimental data indicate that transport properties through the capillary are essentially constant. Evaluation of the detector position for detectors 2 to 8 as function of the detection time of the flow peak (peak S2 in Fig. 3B) revealed a perfect linear relationship $\left(\mathrm{r}^{2}=1.000\right)$ with a slope representing the total flow $(1092$ $\mu \mathrm{m} / \mathrm{s})$ and an intercept $(4.45 \mathrm{~mm})$ indicating the imprecision of detector mounting and/or sample position in relation to the beginning of the capillary. EOF could thus be calculated as being 42.4 $\mu \mathrm{m} / \mathrm{s}$. This value is smaller compared to that observed in Fig. 2B. It is important to mention that according to the EOF simulation model applied, the EOF should be $13.6 \%$ higher compared to the case of Fig. 2. Reasons for this discrepancy were not investigated and the EOF input for the simulation data of Fig. 3A was adjusted to match the experimental EOF as mentioned at the end of Section 3.1.

\subsection{Electrophoretic boundaries formed in a discontinuous buffer system}

After having followed analyte and system peaks in CZE, the dynamics of a discontinuous buffer system in an LPA coated capillary was investigated under constant current and constant voltage conditions. The catholyte (also referred to as LE) was composed of $10 \mathrm{mM} \mathrm{NaOH}$ and $24.6 \mathrm{mM}$ acetic acid (calculated $\mathrm{pH}$ and conductivity of 4.60 and $0.0920 \mathrm{~S} / \mathrm{m}$, respectively) and the anolyte (TE) comprised $10 \mathrm{mM}$ acetic acid (calculated $\mathrm{pH}$ and conductivity of 3.39 and $0.0160 \mathrm{~S} / \mathrm{m}$, respectively). In this configuration, sodium ion of the catholyte is the leading component, $\mathrm{H}_{3} \mathrm{O}^{+}$the terminating ion and acetic acid the counter component $[4,43]$. Upon power application a moving 
steady-state boundary between the LE and the adjusted TE (in this case $30.87 \mathrm{mM}$ HAc with a pH of 3.14 and a conductivity of $0.0283 \mathrm{~S} / \mathrm{m}$ ) is formed which migrates towards the cathode. The migration velocity in absence of any electroosmotic and hydrodynamic buffer flow is given by the relationship $\mathrm{v}=\left(\mathrm{T}_{\mathrm{NA}+} \mathrm{I}\right) /\left(\mathrm{FC}_{\mathrm{Na}+}\right)$ where $\mathrm{T}_{\mathrm{Na}+}$ and $\mathrm{C}_{\mathrm{Na}+}$ refer to the transfer number and concentration of sodium in the catholyte $\left(\mathrm{mol} / \mathrm{m}^{3}\right), \mathrm{F}$ is the Faraday constant $(96487 \mathrm{As} / \mathrm{mol})$ and I the current density $\left(\mathrm{A} / \mathrm{m}^{2}\right)$ [4]. For the given example, $\mathrm{T}_{\mathrm{NA}+}$ equals $0.55\left(\mathrm{~T}=\mathrm{m}_{\mathrm{Na}^{+}} /\left(\mathrm{m}_{\mathrm{Na}^{+}}+\mathrm{m}_{\mathrm{Ac}^{-}}\right)\right.$ where $m_{j}$ are the mobilities of sodium and acetate given in Table 1$)$ and $v=5.70 \times 10^{-7} \mathrm{I}(\mathrm{m} / \mathrm{s})$. Furthermore, at the location of the initial interface between catholyte and anolyte, a non-migrating, diffusing transition between $10 \mathrm{mM}$ and $30.87 \mathrm{mM} \mathrm{HAc}$ is formed $[4,43]$.

GENTRANS predicted conductivity detector data of that system at a sampling rate of $2 \mathrm{~Hz}$ and having the 8 detectors are depicted in Fig. 4A. The selected detector positions were 8.86, 16.71, $23.86,31.29,38.57,45.71,53.86$ and $60.00 \%$ of simulation column length and correspond to those used in the experimental setup corrected for the $5 \%$ initial shift of the sample used in the simulation (cf. Section 2.4). The computer predicted data reveal the sharp decrease between the conductivity of the LE and the adjusted terminator together with the expected advancement of the migrating boundary. Simulations were performed with a $17.5 \mathrm{~cm}$ capillary $(35000$ segments, $\Delta \mathrm{x}=5.0 \mu \mathrm{m})$ at a constant $370 \mathrm{~A} / \mathrm{m}^{2}$ (initial voltage: $871.4 \mathrm{~V}$; voltage at $10 \mathrm{~min}: 2344 \mathrm{~V}$, see data in insert of Fig. 4A) and without imposed flow. A constant EOF of $30 \mu \mathrm{m} / \mathrm{s}$ was assumed to mimic the presence of a small EOF. The simulation could be executed in about $7 \mathrm{~h}$. Simulations with the capillary length used for the experiment together with the applied power would require a much higher mesh and would result in simulation time intervals of up to several weeks. The predicted current for a $50 \mu \mathrm{m}$ ID capillary of $70 \mathrm{~cm}$ length would be $2.91 \mu \mathrm{A}$. This value compares well to that monitored in the experiment (see below). Furthermore, the response of detector $\mathrm{D}_{1}$ shows a sigmoidal conductivity decrease around $3.8 \mathrm{~min}$ (marked with asterisk). This transition comprises the detection of the diffusing boundary which was formed at the location of the initial buffer interface between catholyte and anolyte and does not migrate in the electric field but is transported by buffer flow. Experimental data obtained with the 8-detector setup using a $70 \mathrm{~cm}$ long, $50 \mu \mathrm{m}$ I.D. fused-silica capillary whose inside wall was coated with LPA are depicted in Fig. 4B. Electropherograms of the $8 \mathrm{C}^{4} \mathrm{Ds}$ were normalized to the response of detector 8 such that comparable signal changes were obtained for all detectors. Correction factors for the data of Fig. 4B varied between 1.000 (detector 8) and 1.141 (detector 1) and were used to normalize all other ITP data presented in this work. Data of Fig. 4B were recorded under constant current conditions $(3 \mu \mathrm{A})$ and without application of a hydrodynamic flow. They reveal the sharp decrease between the conductivity of the LE and the 
adjusted terminator together with the advancement of the migrating boundary. During the experiment, the voltage increased from 10.0 to $28.9 \mathrm{kV}$ (insert in Fig. 4B) and the applied power level increased within $10 \mathrm{~min}$ from 0.043 to $0.124 \mathrm{~W} / \mathrm{m}$. The migration velocity of the boundary was calculated to be $870.9 \mu \mathrm{m} / \mathrm{s}$ using the relationship $\mathrm{v}=5.70 \times 10^{-7} \mathrm{I}(\mathrm{m} / \mathrm{s})$ where $\mathrm{I}$ is the current density $\left(\mathrm{A} / \mathrm{m}^{2}\right)$ as is discussed above. The thereby estimated boundary velocity compares well to the mean (SD) net velocity of $960.0 \mu \mathrm{m} / \mathrm{s}(4.0 \mu \mathrm{m} / \mathrm{s})$ elucidated from detector position and detection time of the ITP boundaries, a value which includes migrational transport and buffer flow, i.e. EOF. Furthermore, the responses of detectors $\mathrm{D}_{1}$ (placed at $2.7 \mathrm{~cm}$ ) and $\mathrm{D}_{2}($ at $8.2 \mathrm{~cm}$ ) show a sigmoidal conductivity decrease around 4.0 and $8.9 \mathrm{~min}$, respectively (marked with asterisks). These transitions comprise the detection of the diffusing boundary which was formed at the location of the initial buffer interface between catholyte and anolyte and thus represent buffer flow. As no hydrodynamic flow was applied, these data suggest that there was a small EOF. Based on the data of the first detector, the average velocity was estimated at $112.5 \mu \mathrm{m} / \mathrm{s}$, for the second detector at $153.6 \mu \mathrm{m} / \mathrm{s}$, values which suggest that the EOF is increasing with time. Furthermore, with constant EOF, the sigmoidal transition detected by detector 2 should be significantly broader compared to that of detector 1. The broadening is not observed in the experiment (Fig. 4B) which is in support of the increasing EOF. Not surprisingly, the use of a constant EOF (around $30 \mu \mathrm{m} / \mathrm{s}$ ) for the simulation did not reveal data that matched those monitored experimentally. Within the investigated $10 \mathrm{~min}$ runs, the transition was only predicted for the first detector (Fig. 4A). The transition would have reached the second detector after $10 \mathrm{~min}$ which is not in agreement with the experiment (compare data of Fig. 4A with those of Fig. 4B).

Simulation data for the system of Fig. 4A with ionic strength dependent EOF and otherwise identical conditions are depicted in Fig. 4C. Electroosmosis was calculated as function of ionic strength according to the model first described for fused-silica capillaries which were dynamically double coated with Polybrene and poly(vinylsulfonate) [31]. An electroosmotic mobility of $2.4 \mathrm{x}$ $10^{-9} \mathrm{~m}^{2} / \mathrm{Vs}$ instead of $41.1 \times 10^{-9} \mathrm{~m}^{2} / \mathrm{Vs}$ was used (see Section 3.1 ) and the predicted EOF increased from 18.04 to $67.47 \mu \mathrm{m} / \mathrm{s}$ within 10 min of power application (insert in Fig. 4C). Using these conditions, the time scales of predicted and experimental electropherograms became nearly identical and predicted electropherograms, including the positions of the sigmoidal flow marker transitions of detectors 1 and 2, were qualitatively in agreement with those monitored experimentally. The simulation could be executed in about 9 hours. The EOF model used predicts a higher pumping rate in regions of lower ionic strength and higher electric field. The ionic strength of adjusted TE and the TE were predicted to be 0.73 and $0.41 \mathrm{mM}$, respectively, values that are much smaller compared to the $10.02 \mathrm{mM}$ ionic strength of the LE. Furthermore, the electric field in 
these zones is much higher compared to that within the LE. Thus, the prediction of a steadily increasing EOF (insert in Fig. 4C) makes sense. In ITP, the LE is gradually replaced by adjusted TE and TE and the used EOF model appears to take into account that fact in a reasonable way for an LPA coated capillary.

The data depicted in Fig. 5 were obtained under constant voltage conditions, at $1.5 \mathrm{kV}$ for the simulation data (Fig. 5A) and at $20 \mathrm{kV}$ in the experiment (Fig. 5B). The same boundary was observed. However, due to the decreasing current (see insert in Fig. 5B), it migrated with gradually decreasing velocity. Using the same configuration and EOF model as for Fig. 4C, matching simulation data were obtained (compare data of Fig. 5A and 5B). Within the 10 min time interval, the predicted current density decreased from 637.00 to $243.18 \mathrm{~A} / \mathrm{m}^{2}$ (insert in Fig. 5A). The predicted current for a $50 \mu \mathrm{m}$ ID capillary of $70 \mathrm{~cm}$ length would accordingly decrease from 5.00 to $2.39 \mu \mathrm{A}$. This change is comparable to that observed experimentally $(4.41-2.32 \mu \mathrm{A})$. The predicted EOF increased from 22.78 to $29.64 \mu \mathrm{m} / \mathrm{s}$ (insert in Fig. 5A). This change is smaller compared to that predicted under constant current conditions.

It was interesting to find that predicted conductivity changes are somewhat different to those monitored with contactless conductivity detection. This is best seen with the sigmoidal transitions predicted for and detected with detectors 1 (Fig. 4 and Fig. 5) and 2 (Fig. 5) which is characterized with a conductivity change of $0.0123 \mathrm{~S} / \mathrm{m}$ in relation to the $0.0637 \mathrm{~S} / \mathrm{m}$ change across the migrating ITP boundary. This indicates that the conductivity change across the ITP boundary is predicted to be 5.2 times larger compared to the change across the sigmoidal transition. The corresponding signal ratio in the experiments is about 8.5. Efforts in using input values for simulation that were corrected for ionic strength (pKa and mobility values, as described in [29]) revealed an even lower ratio of 4.4 and did not provide an explanation of the discrepancy between simulation and experiment. Thus, it is assumed that contactless conductivity detection as used in this work is providing a non-linear response for the conductivity changes encountered in the ITP boundary example. More work is required to assess this difference.

\subsection{Isotachophoretic sample zone followed by array detection}

After studying the behavior of a migrating and a non-migrating boundary of a discontinuous buffer system in a LPA coated capillary, work was directed towards the formation of an ITP sample zone. A sample composed of +NPE and -NPE (2.85 mM each) was applied to the same LE and TE system as described in Section 3.3. It represents the same sample as was used in a separate project dealing with the study of the stereoselective ITP separation process. Without a suitable chiral selector in the LE, the two analytes cannot be separated by ITP and thus form a homogeneous 
cationic sample zone between the LE and the adjusted TE. Fig. 6A depicts computer simulated data at constant current density of $370 \mathrm{~A} / \mathrm{m}^{2}$ and using the same EOF model as for Fig. 4C. The initial sample zone length was $2.5 \%$ of column length with a boundary width of $0.02 \%$ (for initial component distribution see lower insert in Fig. 6A). The sample zone is demarcated with sharp conductivity changes and migrates along the capillary as ITP zone of constant length and with an electrophoretic transport rate of $211 \mu \mathrm{m} / \mathrm{s}$ calculated with $\mathrm{v}=5.70 \times 10^{-7} \mathrm{I}(\mathrm{m} / \mathrm{s})$ as described in Section $3.3[4,43,44]$. The EOF is predicted to increase from 33.21 to $77.77 \mu \mathrm{m} / \mathrm{s}$ within the $10 \mathrm{~min}$ time interval (dashed line graph in upper insert of Fig. 6A) and is smaller compared to the electrophoretic transport. Thus, the net transport rate is slightly increasing with time. Experimental results obtained at a constant current of $3 \mu \mathrm{A}$ are depicted in Fig. 6B. The sample was introduced by application of 1.6 psi for $13 \mathrm{~s}$, thereby occupying a capillary segment of about $2.5 \%$ of capillary length (calculated with CE Expert Lite software). Experimentally monitored detector profiles of detectors 2 to 7 correspond well with those predicted by GENTRANS. For the chosen conditions under a constant current density, the voltage is predicted to increase in a similar fashion as was predicted by simulation (compare voltage graphs in upper insert of Fig. 6A with insert of Fig. 6B).

For detector 1 and the transition marked with asterisk detected with detector 2, the predicted detector profiles are somewhat different compared to those monitored experimentally. With detection at an earlier position than that of detector 1 provided a predicted detector signal which compares better to that monitored with detector 1 . This is shown with the dashed line graph in Fig. $6 \mathrm{~A}$ which corresponds to a detector placed at 2.0 instead of $2.7 \mathrm{~cm}$ of the $70 \mathrm{~cm}$ long capillary. At this location, however, the first detector could not be placed in the used setup. Thus, other sources with impact on detector profiles at early column locations were investigated. Transient states occurring prior to the formation of the ITP zone are dependent on the initial composition of the sample. Broader initial boundaries between sample and its surrounding electrolytes (LE and TE on cathodic and anodic side, respectively) were found to have an impact on the predicted detector signal of the first detector and the buffer transition of detector 2 marked with an asterisk in Fig. 6. This is illustrated with the data of Fig. $6 \mathrm{C}$ which were obtained with very diffuse initial sample boundaries (lower inset in Fig. 6C). Better agreement between experimental and simulation data for detector 1 and for the buffer transition of detector 2 was thereby found. These findings suggest that the initial sample plug produced by the SIA setup does not comprise sharp boundaries between sample and the two electrode solutions. More work is required to study this phenomenon.

\section{Concluding remarks}


Data obtained with dynamic simulation and the laboratory made CE system featuring an array of 8 $\mathrm{C}^{4}$ Ds along an LPA coated fused-silica capillary were found to be in agreement for various configurations, including CZE with a small constant EOF and ITP with a varying EOF. The techniques provide means for quickly investigating the zone electrophoretic separability of components and the required capillary length for that task under specified experimental conditions. Furthermore, in the case of CZE, the discussed data demonstrate that system peaks and interferences between system peaks and analytes can be properly recognized. The same would be true for separations and analyses based on electrokinetic capillary chromatography, including those using monolithic columns for which contactless conductivity detection was demonstrated to be feasible [45]. All experiments were made in a setup which permits positioning of the first $\mathrm{C}^{4} \mathrm{D}$ as close as $2.7 \mathrm{~cm}$ from the sample inlet such that basic phenomena, including the visualization of EOF and transient patterns in ITP, could be explored. Data obtained demonstrate that modelling alone is not sufficient for cases without realistic input parameters. This was found to be true for the prediction of (i) EOF in an LPA coated capillary under ITP conditions and (ii) formed transient configurations around the sampling compartment of the capillary. Gathering of simulation and experimental data led to the elucidation of the temporal behavior of EOF and, for the first time, finding of a reasonable model to estimate the EOF in an LPA coated capillary. EOF can be estimated with an ionic strength dependent model similar to that previously used to describe EOF in fused-silica capillaries dynamically double coated with Polybrene and poly(vinylsulfonate) and is not constant for configurations with discontinuous buffer systems such as ITP. All data demonstrate that it is beneficial to have multiple detectors along $\mathrm{CE}$ columns for studying the separation processes. A detector array can quickly give a lot of information useful in the development of an application or, as in the cases reported here, in the improvement of input parameters for simulation. Dynamic simulation, on the other hand, is rather slow but provides insight into basic phenomena of ion transport and EOF. Furthermore, a detector position close to the sampling compartment provides information that can be used for the improvement of the instrumental setup. Dynamic computer simulation and CE with an array of $\mathrm{C}^{4} \mathrm{Ds}$ are currently used to follow isotachophoretic enantiomer separations and results will be reported in a second contribution. The same technology will also be employed to study formation and migration behavior of a variety of other electrophoretic boundaries in discontinuous buffer systems, including those associated with IEF.

\section{Acknowledgements}

This work was sponsored by the Swiss National Science Foundation (Grants 315230-146161 200020-149068). 


\section{Conflicts of interest}

The authors have declared no conflict of interest. 


\section{References}

[1] Stojkovic, M., Koenka, I.J., Thormann, W., Hauser, P.C., Electrophoresis 2014, 35, 482-486.

[2] Thormann, W., Arn, D., Schumacher, E., Electrophoresis 1984, 5, 323-337.

[3] Thormann, W., Arn, D., Schumacher, E., Sep. Sci. Technol. 1984, 19, 995-1011.

[4] Thormann, W., Arn, D., Schumacher, E., Electrophoresis 1985, 6, 10-18.

[5] Thormann, W., Mosher, R. A., Bier, M., J. Chromatogr. 1986, 351, 17-29.

[6] Thormann, W., Egen, N. B., Mosher, R. A., Bier, M., J. Biochem. Biophys. Methods 1985, 11, 287293.

[7] Kubáň, P., Hauser, P. C., Electrophoresis 2013, 34, 55-69.

[8] Mai, T. D., Hauser, P. C., Chem. Record 2012, 12, 106-113.

[9] Coltro, W. K. T., Lima, R. S., Segato, T. P., Carrilho, E., de Jesus, D. P., do Lago, C. L., da Silva, J. A. F., Anal. Methods 2012, 4, 25-33.

[10] Kubáň, P., Hauser, P. C., Electrophoresis 2011, 32, 30-42.

[11] Kubán̆, P., Hauser, P. C., Electrophoresis 2009, 30, 176-188.

[12] Trojanowicz, M., Anal. Chim. Acta 2009, 653, 36-58.

[13] Gaš, B., Demjanenko, M., Vacík, J., J. Chromatogr. 1980, 192, 253-257.

[14] Hjertén, S., Chromatogr. Rev. 1967, 9, 122-219.

[15] Hirokawa, T., Nakahara, K., Kiso, Y., J. Chromatogr. 1989, 463, 39-49.

[16] Wang, T., Hartwick, R.A., Anal. Chem. 1992, 64, 1745-1747.

[17] Beale, S.C., Sudmeier, S.J., Anal. Chem. 1995, 67, 3367-3371.

[18] Wu, X.-Z., Huang, T., Liu, Z., Pawliszyn, J., Trends Anal. Chem. 2005, 24, 369-382.

[19] Bier, M., Palusinski, O.A., Mosher, R.A., Saville, D.A., Science 1983, 219, 1281-1287.

[20] Mosher, R. A., Saville, D. A., Thormann, W., The Dynamics of Electrophoresis, VCH Publishers, Weinheim 1992.

[21] Thormann, W., Zhang, C.-X., Caslavska, J., Gebauer, P., Mosher, R.A., Anal. Chem. 1998, 70, 549562.

[22] Breadmore, M.C., Kwan, H.Y., Caslavska, J., Thormann, W., Electrophoresis 2012, 33, 958-969.

[23] Hruška, V., Jaroš, M., Gaš, B., Electrophoresis 2006, 27, 984-991.

[24] Hruška, V., Beneš, M., Svobodová, J., Zusková, I., Gaš, B., Electrophoresis 2012, 33, 938-947.

[25] Bercovici, M., Lele, S.K., Santiago, J.G., J. Chromatogr. A 2009, 1216, 1008-1018.

[26] Thormann, W., Caslavska, J., Breadmore, M.C., Mosher, R.A., Electrophoresis 2009, 30, S16-S26.

[27] Thormann, W., Breadmore, M. C., Caslavska, J., Mosher, R. A., Electrophoresis 2010, 31, 726-754.

[28] Mosher, R.A., Breadmore, M.C., Thormann, W., Electrophoresis 2011, 32, 532-541.

[29] Caslavska, J., Mosher, R.A., Thormann, W., Electrophoresis 2015, 36, 1529-1538.

[30] Caslavska, J., Thormann, W., J. Microcol. Sep. 2001, 13, 69-83.

[31] Caslavska, J., Thormann, W., Electrophoresis 2006, 27, 4618-4630.

[32] Thormann, W, Caslavaska, J., Mosher, R.A., J. Chromatogr. A 2007, 1155, 154-163.

[33] Mai, T. D., Schmid, S., Müller, B., Hauser, P. C., Anal. Chim. Acta 2010, 665, 1-6.

[34] Mai, T. D., Hauser, P. C., Talanta 2011, 84, 1228-1233.

[35] Mai, T. D., Hauser, P. C., Electrophoresis 2011, 32, 3000-3007.

[36] Stojkovic, M., Mai, T. D., Hauser, P., Anal. Chim. Acta 2013, 787, 254-259.

[37] Mai, T. D., Hauser, P. C., J. Chromatogr. A 2012, 1267, 266-272.

[38] Koenka, I.J., Sáiz, J., Hauser, P.C., Comput. Phys. Commun. 2014, 185, 2724-2729.

[39] Koenka, I.J., Sáiz, J., Hauser, P.C., Chimia 2015, 69, 172-175.

[40] Gaš, B., Jaroš, M., Hruška, V., Zusková, M.S., Štědrý, M., LC-GC Eur. 2005, 18, 282-288.

[41] Nakatani, M., Shibukawa, A., Nakagawa, T., Electrophoresis 1995, 16, 1451-1456.

[42] Gaš, B., Hruška, V., Dittmann, M., Bek, F., Witt, K., J. Sep. Sci. 2007, 30, 1435-1445.

[43] Boček, P., Gebauer, P., Deml, M., J. Chromatogr. 1981, 217, 209-224.

[44] Snopek, J., Jelínek, I., Smolková-Keulemansová, E., J. Chromatogr. 1988, 438, 211-218.

[45] Mai, T.D., Pham, H.V., Hauser, P.C., Anal. Chim. Acta 2009, 653, 228-233. 


\section{Legends}

Figure 1: Schematic representation of the CE set-up with a SIA manifold and a separation capillary with eight contactless conductivity detectors. Key: HV: high voltage, S: solution, D: detector, W: waste, P: pressure regulator, SP: syringe pump, I: injector interface, LD: loading coil.

Figure 2: (A) Simulated and (B) experimental conductivity electropherograms of detectors $D_{1}$ to $D_{8}$ (presented with a y-scale offset) for a zone electrophoretic separation of two cations and two anions in an LPA coated fused-silica capillary using a BGE composed of $20 \mathrm{mM}$ formic acid and $10 \mathrm{mM}$ $\mathrm{NaOH}$ under conditions of constant voltage and a constant imposed buffer flow towards the cathode. The sample ( $1 \mathrm{mM}$ of each component in BGE) was applied as a $1 \%$ pulse at the anodic capillary end. For information about applied voltage and flow see text. Key of peaks: A: arginine, B: benzoic acid, G: glycolic acid, T: tryptamine, S1: cationic migrating system peak, S2: system peak with no migration (flow marker).

Figure 3: (A) Simulated and (B) experimental conductivity electropherograms of detectors $D_{1}$ to $D_{8}$ (presented with a y-scale offset) for a zone electrophoretic separation of the same sample in a BGE composed of $10 \mathrm{mM}$ formic acid and $5 \mathrm{mM} \mathrm{NaOH}$ and otherwise identical conditions as in Fig. 2. Key of peaks as for Fig. 2.

Figure 4: (A,C) Simulated and (B) experimental conductivity electropherograms of detectors $\mathrm{D}_{1}$ to $\mathrm{D}_{8}$ for detection of a cationic ITP boundary between sodium as leading component and $\mathrm{H}_{3} \mathrm{O}^{+}$as terminating component under constant current conditions in an LPA coated fused-silica capillary. The LE (catholyte) was composed of $10 \mathrm{mM} \mathrm{NaOH}$ and $24.6 \mathrm{mM}$ acetic acid and the TE (anolyte) was $10 \mathrm{mM}$ acetic acid. Simulations were performed with (A) a constant EOF and (C) an ionic strength dependent EOF. For details refer to text. The inserts in panels $\mathrm{A}$ and $\mathrm{C}$ comprise computer predicted temporal behavior of voltage $\mathrm{V}$ (V, left $\mathrm{y}$-axis scale), current density I (A/m $\mathrm{m}^{2}$, left y-axis) and EOF $(\mu \mathrm{m} / \mathrm{s}$, right $\mathrm{y}$-axis scale). The insert in panel B represents recorded current and voltage. The asterisks mark the transitions between adjusted TE and TE (EOF marker).

Figure 5: (A) Simulated and (B) experimental conductivity electropherograms of detectors $D_{1}$ to $D_{8}$ for detection of a cationic ITP boundary between sodium as leading component and $\mathrm{H}_{3} \mathrm{O}^{+}$as terminating component under constant voltage conditions. The configuration was the same as for Fig. 4. Simulations were performed with an ionic strength dependent EOF as for Fig. 4C. For details refer to text. The insert in panel A comprises computer predicted temporal behavior of voltage $\mathrm{V}\left(\mathrm{V}\right.$, left $\mathrm{y}$-axis scale), current density I ( $\mathrm{A} / \mathrm{m}^{2}$, left $\mathrm{y}$-axis) and $\mathrm{EOF}(\mu \mathrm{m} / \mathrm{s}$, right $\mathrm{y}$-axis scale). The insert in panel $\mathrm{B}$ represents recorded current and voltage. The asterisks mark the transitions between adjusted TE and TE (EOF marker).

Figure 6: (A,C) Simulated and (B) experimental conductivity electropherograms of detectors $D_{1}$ to $\mathrm{D}_{8}$ for detection of a cationic ITP zone formed by NPE between sodium as leading component and $\mathrm{H}_{3} \mathrm{O}^{+}$as terminating component under constant current conditions. Simulations were performed with an ionic strength dependent EOF as was used for Fig. 4C having (A) sample boundary widths of $0.02 \%$ shown as lower insert of panel $\mathrm{A}$ with a $\mathrm{mM}$ y-axis unit and $(\mathrm{C})$ very diffuse boundaries which are depicted in the lower insert of panel $\mathrm{C}$. The dashed line graphs in panel A and $\mathrm{C}$ were obtained for a position of detector 1 corresponding of 2.0 instead of $2.7 \mathrm{~cm}$. Upper inserts in panels $\mathrm{A}$ and $\mathrm{C}$ depict the temporal behavior of voltage $\mathrm{V}$ (V, left y-axis scale), current density $\mathrm{I}\left(\mathrm{A} / \mathrm{m}^{2}\right.$, left $y$-axis scale) and EOF as dashed line graph $(\mu \mathrm{m} / \mathrm{s}$, right $\mathrm{y}$-axis scale). For details refer to text. The asterisks mark the transitions between adjusted TE and TE. Other conditions as for Fig. 4. 
Table 1: Physico-chemical input parameters used for simulation ${ }^{\text {a) }}$

\begin{tabular}{|l|l|l|}
\hline Compound & pKa & $\begin{array}{l}\text { Mobility } \\
\left(\mathbf{x ~ 1 0}^{-\mathbf{8}} \mathbf{~ m}^{\mathbf{2}} / \mathbf{V s} \mathbf{~}\right)\end{array}$ \\
\hline Acetic acid & 4.75 & 4.24 \\
\hline Formic acid & 3.75 & 5.66 \\
\hline Benzoic acid & 4.20 & 3.36 \\
\hline Glycolic acid & 3.89 & 4.24 \\
\hline Tryptamine & 9.25 & 3.00 \\
\hline Arginine & $8.92,12.48$ & $2.69^{\mathrm{b})}$ \\
\hline Norpseudoephedrine & 8.9 & $2.65^{\mathrm{c})}$ \\
\hline $\mathrm{Na}^{+}$ & - & 5.19 \\
\hline
\end{tabular}

a) From data base of PeakMaster and SIMUL5.

b) Equal mobilities were assigned to all species.

c) Estimated value 


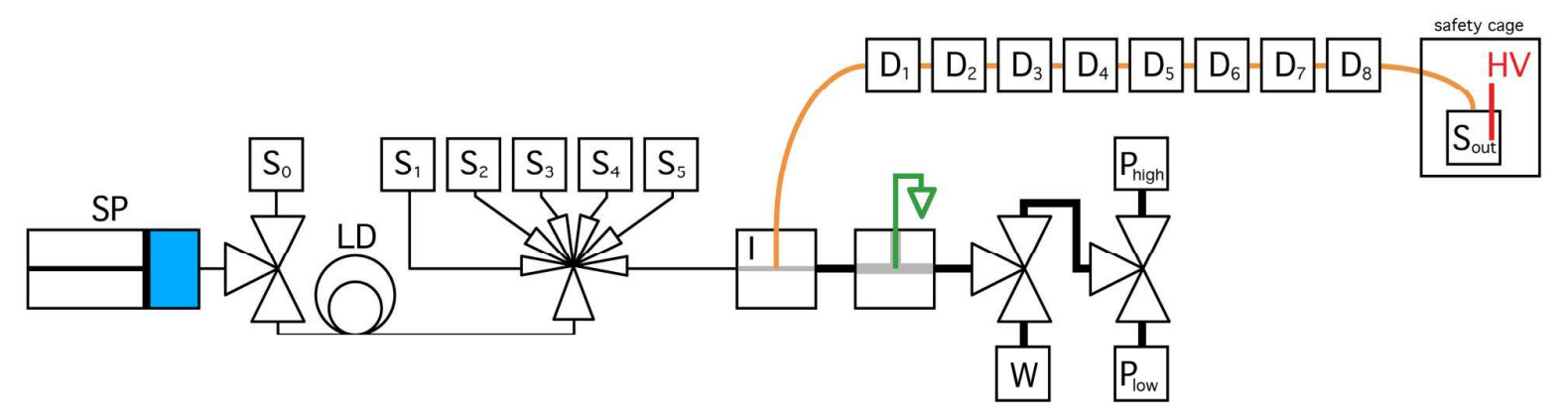

Figure 1 

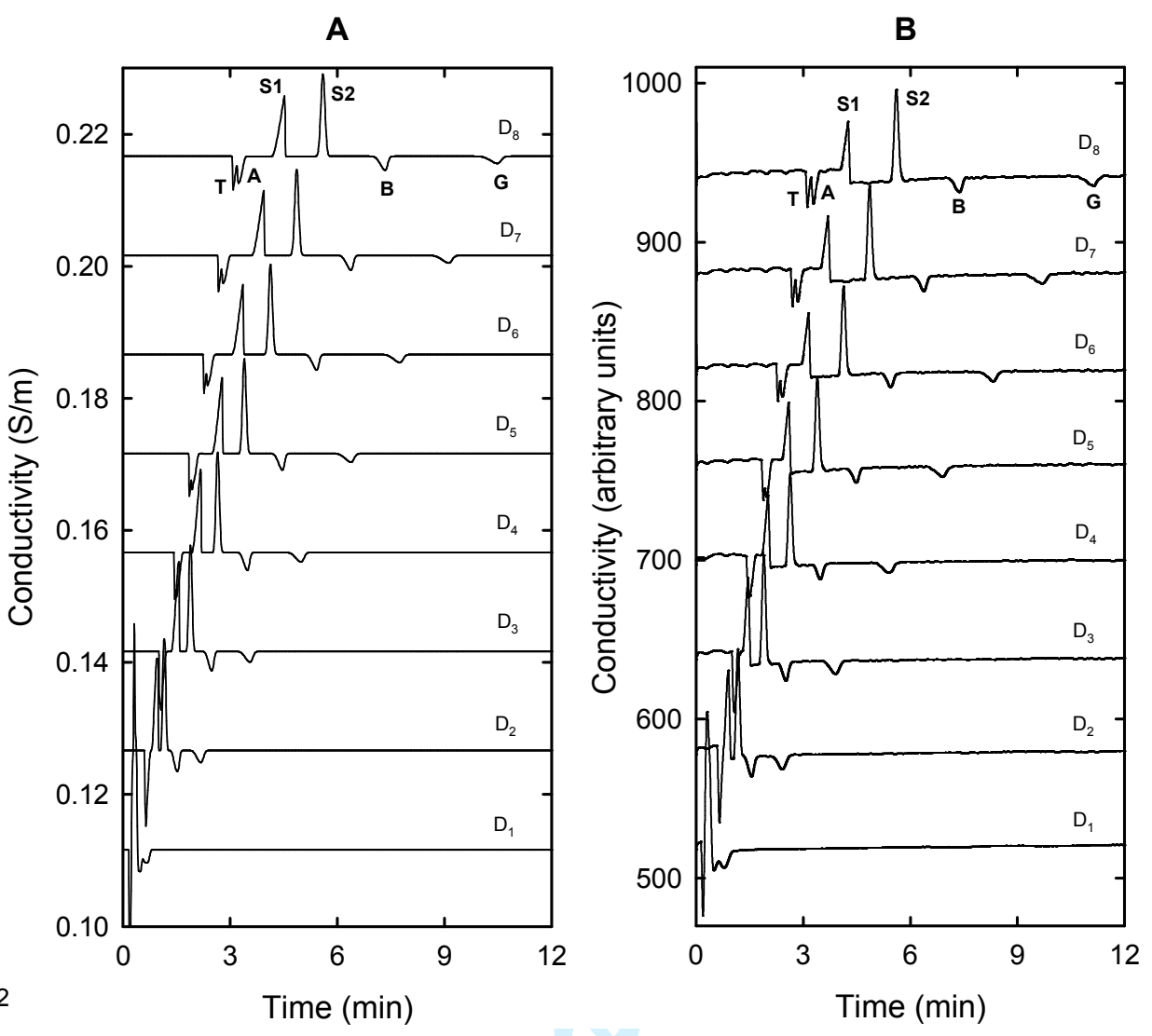

Figure 2

29

30

31

32

33

34

35

36

37

38

39

40

41

42

43

44

45

46

47

48

49

50

51

52

53

54

55

56

57

58

59 

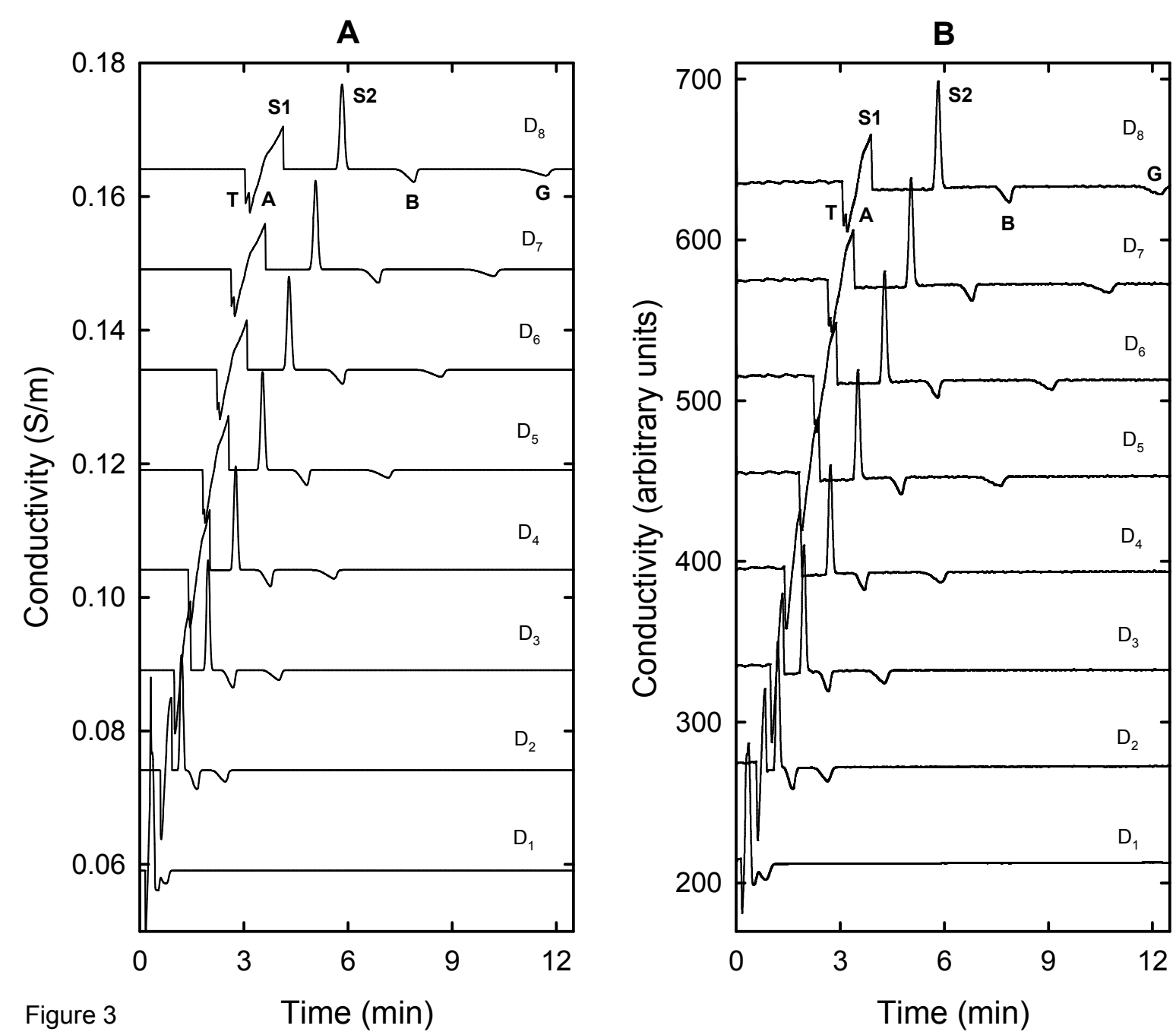

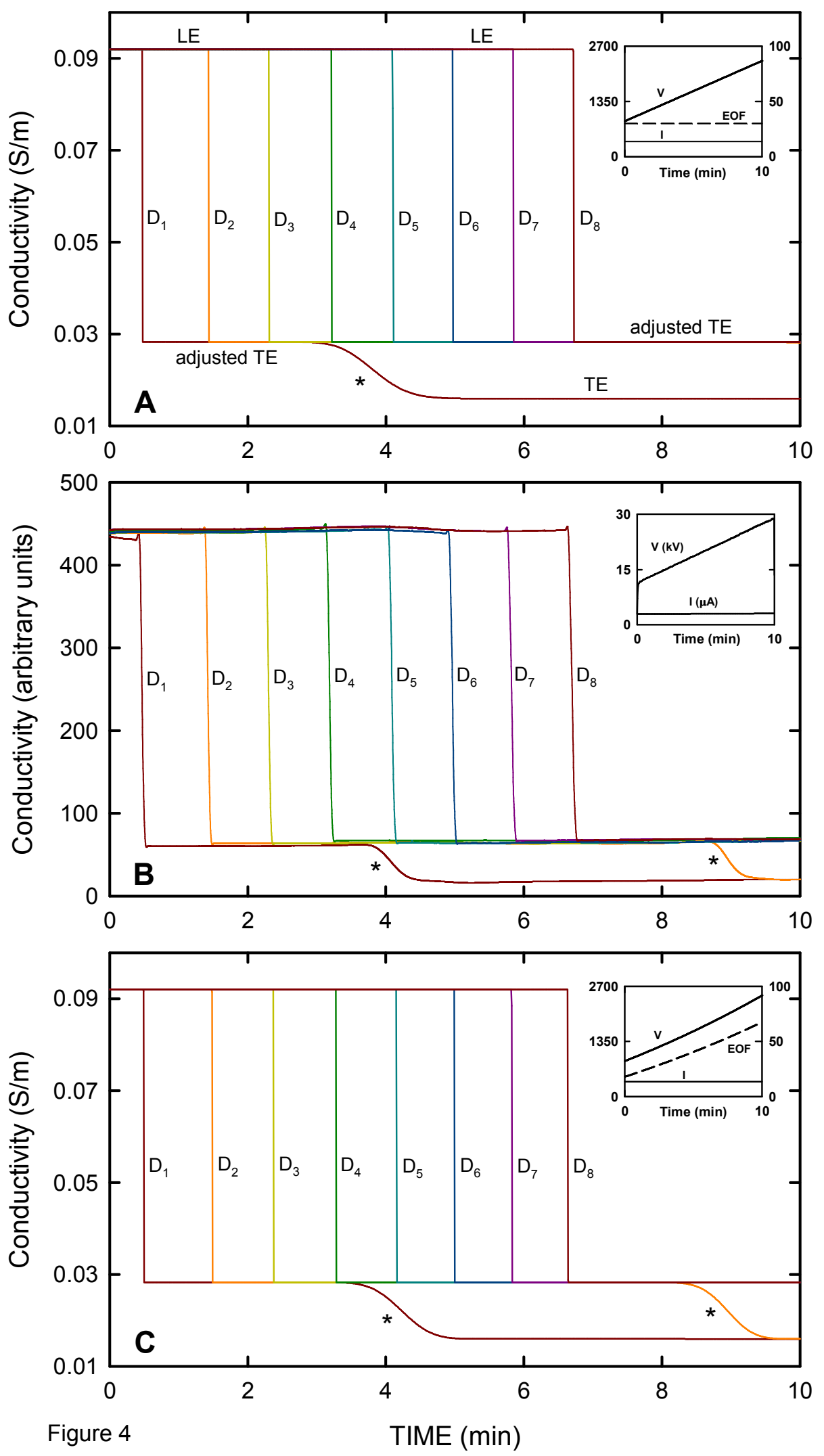

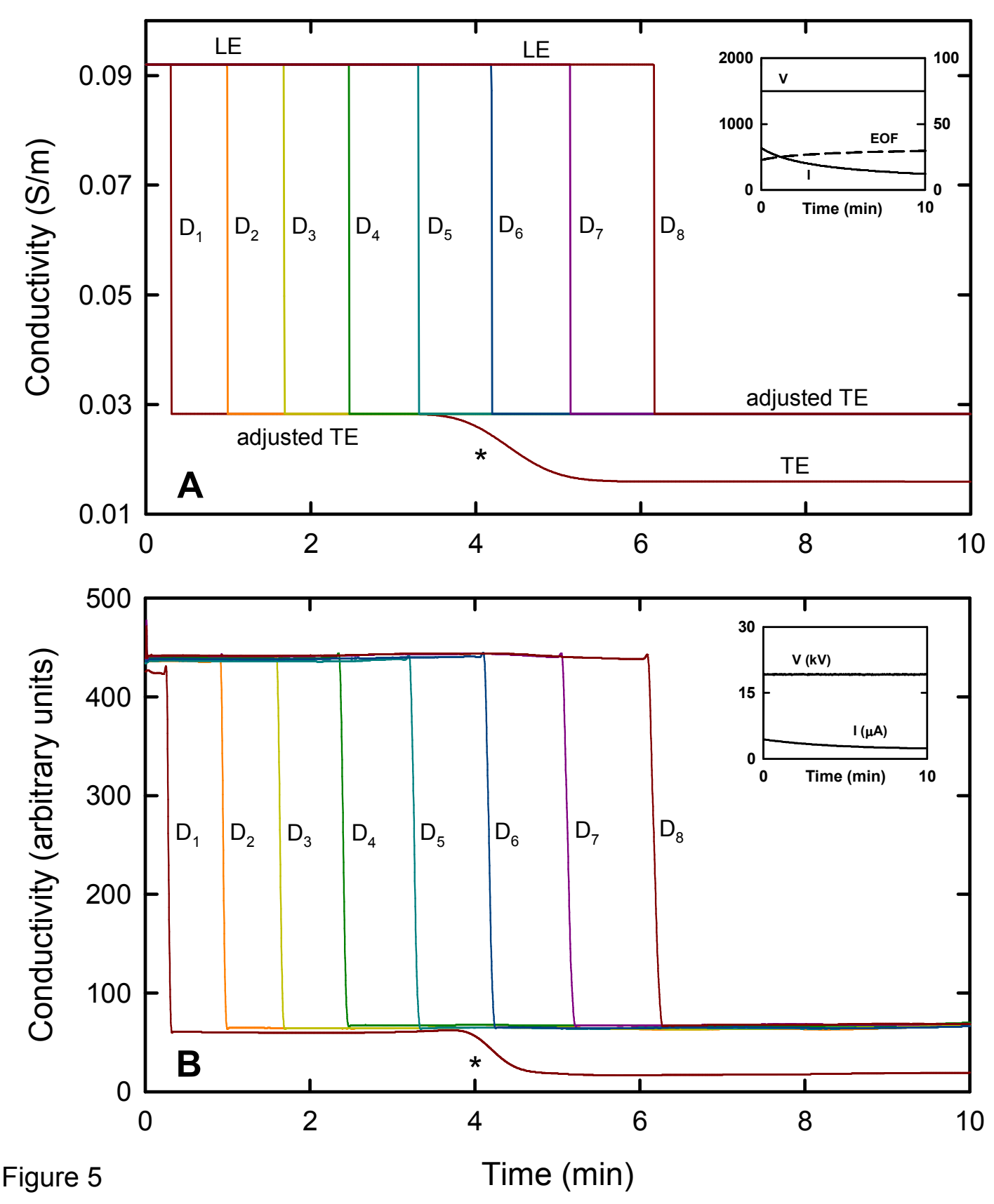

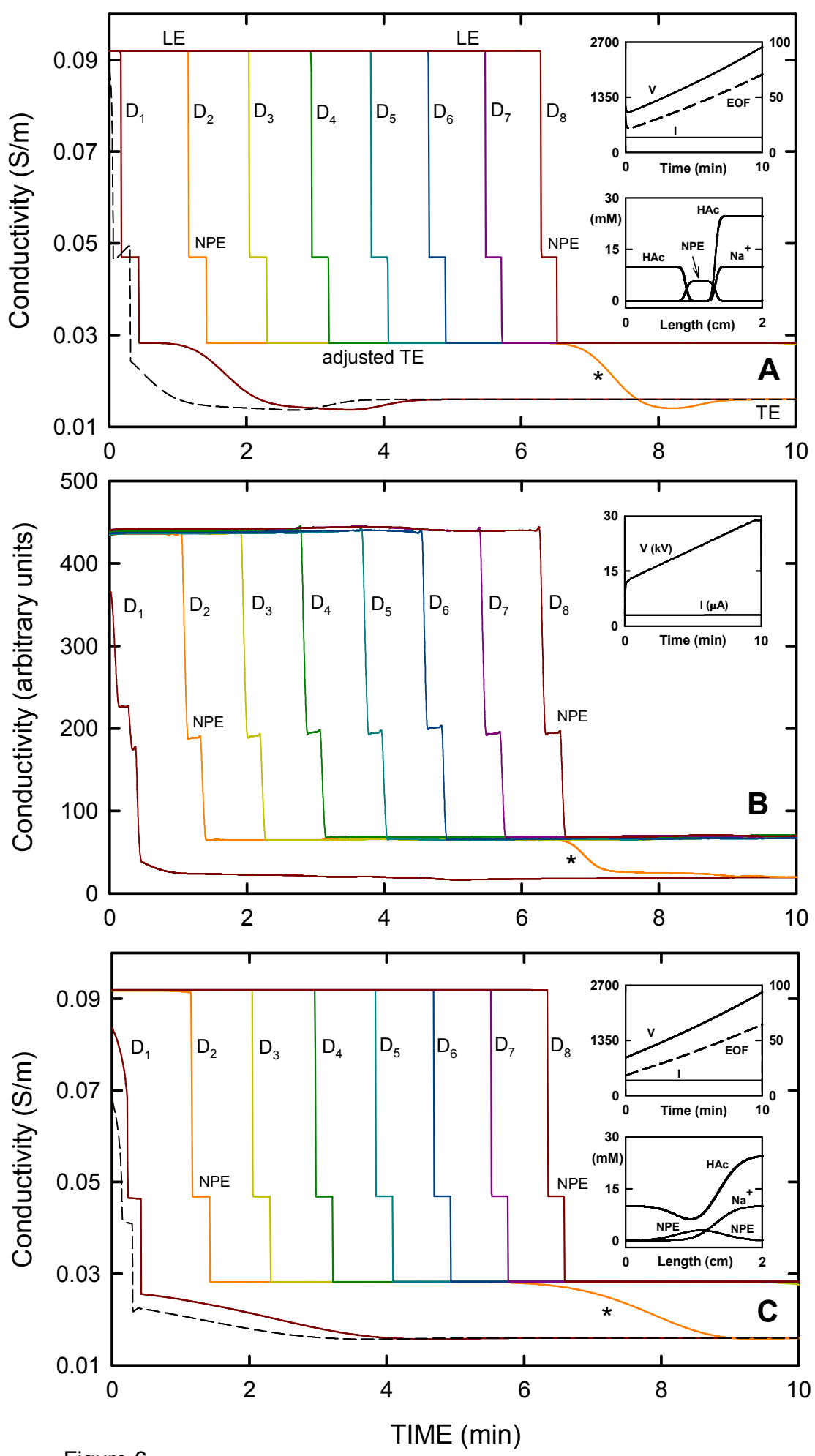

Figure 6 